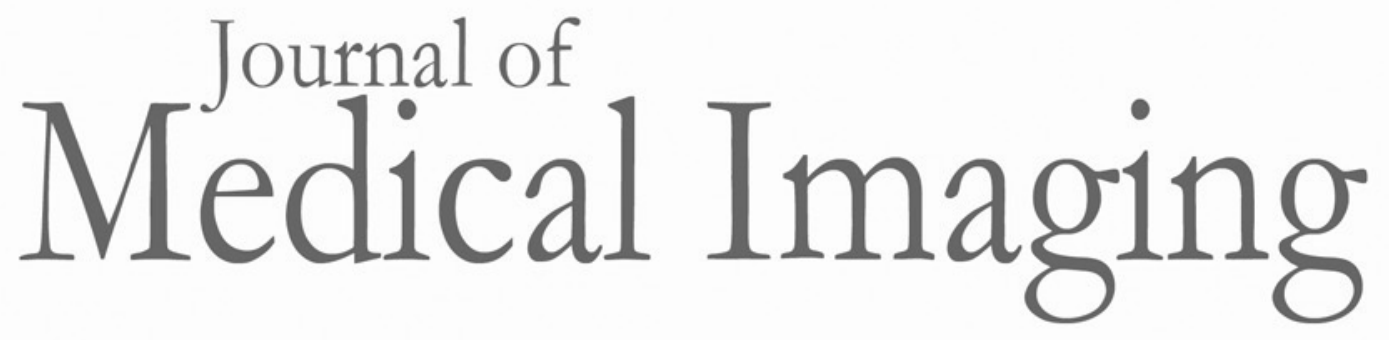

Medicallmaging.SPIEDigitalLibrary.org

\title{
Navigation of guidewires and catheters in the body during intervention procedures: a review of computer-based models
}

Hoda Sharei

Tanja Alderliesten

John J. van den Dobbelsteen

Jenny Dankelman 


\title{
Navigation of guidewires and catheters in the body during intervention procedures: a review of computer-based models
}

\author{
Hoda Sharei, ${ }^{a, \star}$ Tanja Alderliesten, ${ }^{b}$ John J. van den Dobbelsteen, ${ }^{a}$ and Jenny Dankelman ${ }^{a}$ \\ ${ }^{a}$ Delft University of Technology, Department of Biomechanical Engineering, Faculty of Mechanical, Maritime and Materials Engineering, \\ Delft, The Netherlands \\ ${ }^{\mathrm{b}}$ Academic Medical Center, Department of Radiation Oncology, Amsterdam, The Netherlands
}

\begin{abstract}
Guidewires and catheters are used during minimally invasive interventional procedures to traverse in vascular system and access the desired position. Computer models are increasingly being used to predict the behavior of these instruments. This information can be used to choose the right instrument for each case and increase the success rate of the procedure. Moreover, a designer can test the performance of instruments before the manufacturing phase. A precise model of the instrument is also useful for a training simulator. Therefore, to identify the strengths and weaknesses of different approaches used to model guidewires and catheters, a literature review of the existing techniques has been performed. The literature search was carried out in Google Scholar and Web of Science and limited to English for the period 1960 to 2017. For a computer model to be used in practice, it should be sufficiently realistic and, for some applications, real time. Therefore, we compared different modeling techniques with regard to these requirements, and the purposes of these models are reviewed. Important factors that influence the interaction between the instruments and the vascular wall are discussed. Finally, different ways used to evaluate and validate the models are described. We classified the developed models based on their formulation into finite-element method (FEM), mass-spring model (MSM), and rigid multibody links. Despite its numerical stability, FEM requires a very high computational effort. On the other hand, MSM is faster but there is a risk of numerical instability. The rigid multibody links method has a simple structure and is easy to implement. However, as the length of the instrument is increased, the model becomes slower. For the level of realism of the simulation, friction and collision were incorporated as the most influential forces applied to the instrument during the propagation within a vascular system. To evaluate the accuracy, most of the studies compared the simulation results with the outcome of physical experiments on a variety of phantom models, and only a limited number of studies have done face validity. Although a subset of the validated models is considered to be sufficiently accurate for the specific task for which they were developed and, therefore, are already being used in practice, these models are still under an ongoing development for improvement. Realism and computation time are two important requirements in catheter and guidewire modeling; however, the reviewed studies made a trade-off depending on the purpose of their model. Moreover, due to the complexity of the interaction with the vascular system, some assumptions have been made regarding the properties of both instruments and vascular system. Some validation studies have been reported but without a consistent experimental methodology. (- The Authors. Published by SPIE under a Creative Commons Attribution 3.0 Unported License. Distribution or reproduction of this work in whole or in part requires full attribution of the original publication, including its DOI. [DOI: 10.1117/1.JMI.5.1.010902]
\end{abstract}

Keywords: guidewire; catheter; modeling; simulation; training; virtual reality; vascular phantom.

Paper 17289VR received Sep. 19, 2017; accepted for publication Jan. 4, 2018; published online Jan. 29, 2018.

\section{Introduction}

Endovascular interventions include a variety of techniques that give access to the vascular system through small incisions. The access is mainly via guidewires and catheters. Despite the advantages of these procedures, such as decreased surgical trauma and accelerated recovery, ${ }^{1-3}$ new challenges are imposed on specialists. For example, they lose the direct access and the visual feedback and instead they have to manipulate the instrument (i.e., the guidewire and the catheter) from outside the body by applying a translation and/or rotation motion at its proximal side.

Traditionally, the way to learn these skills is by iterative learning on a patient. However, this incorporates a high risk

*Address all correspondence to: Hoda Sharei, E-mail: h.sharei-amarghan@ tudelft.nl for the patient and is also time-consuming. Another way is using cadavers or live animals. These methods are expensive and neither of them completely resembles an actual human vascular system. Employing phantoms is another emerging way to practice the new skills; however, the trainee is restricted to limited possible geometries. An additional drawback of the mentioned training methods is the exposure to x-ray during the training since the visual feedback is provided by $\mathrm{x}$-ray imaging. Consequently, there is no single method that satisfies all the requirements. ${ }^{4,5}$

Another complicating factor is that each instrument has different mechanical properties, and a high degree of expertise is required to select the best one for a particular case. Until now, selecting the instrument has been often based on specialist's experience, which does not always result in a successful procedure. $^{6}$ 
Recently, the use of computer models to predict the behavior of guidewire and catheter has become increasingly popular., The purposes of these models include training simulator, preintervention planning (specifically evaluating the performance of an instrument for a specific procedure), and designing instruments.

Despite the growing trend toward computer models, a comprehensive review of different modeling approaches has not yet been performed. Therefore, this article has four goals: (1) to introduce the purposes of guidewire and catheter modeling, (2) to survey different approaches used for instrument modeling and identify their strengths and weaknesses, (3) to study the important factors that affect the interaction between the instrument and the vascular wall, and (4) finally, to review the different strategies used to validate the simulation. We will outline the key areas where future research can improve.

\section{Review Method}

To obtain a comprehensive overview of guidewire and catheter models developed in different studies, we first used Google Scholar as the main search engine and then Web of Science for supplementary information. The keywords were "guidewire," "catheter," "modeling," "simulation," "training," "virtual reality," and "vascular phantom." Boolean operators (AND, OR, and NOT) were used to combine search terms, and wildcards were applied to deal with spelling variations. Next, criteria for exclusion/inclusion of publications were set, and articles were selected based on their title. Then, the abstract of each selected article was fully read, and the article was either included or excluded based on the relevance and applicability of the content. Finally, to complete the literature search, extra resources from citations and references of the included articles were screened and added when appropriate. In case of duplicate publications, the most recent was included.

\section{Results}

\subsection{Purposes of Computer Models}

In guidewire/catheter modeling, researchers have focused on purposes such as training, preintervention planning, and designing instruments. Although achieving these might overlap (Fig. 1), we will review each one separately.

\subsubsection{Training}

Simulation-based training is a virtual environment, which helps the specialists to learn complex skills and new catheterization techniques by trial and error without risking patient safety. ${ }^{7,8}$ In this way, the training becomes more efficient and cost-effective compared to traditional training methods (e.g., using human cadavers and animals). Researchers follow two main approaches: (1) developing a model while focusing on the modeling techniques ${ }^{9-32}$ and (2) investigating the effectivity and the necessity of using these simulations for training purposes. . $^{7,83-36}$

\subsubsection{Preintervention planning}

A simulation can also be used to evaluate the performance of an instrument for a specific anatomy prior to the procedure. This information assists the specialist to select an instrument with the proper mechanical properties and, as a result, increases the success rate of a procedure in accessing the target location. The research done in this field either focuses on

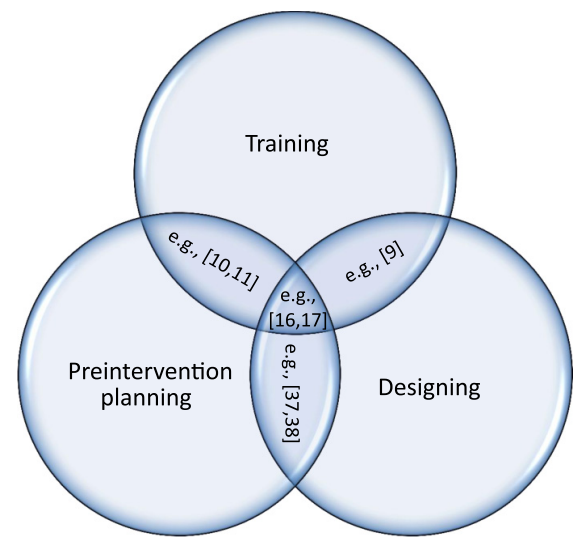

Fig. 1 Purposes of a guidewire/catheter model.

catheter ${ }^{10,11,16,17,20,37,38}$ or on guidewire selection. ${ }^{39-41}$ However, in practice, the instrument selection procedure is still based on the specialist's experience, which is subjective rather than objective.

\subsubsection{Designing instruments}

Design optimization of instruments by predicting their behavior inside the body is another purpose of the computer models, ${ }^{9,10,16,17,37,38,42,43}$ and they are used to test different materials and structures for such instruments and to assess their performance to achieve optimal design. Both numerical (e.g., Ref. 38) and analytical (e.g., Ref. 9) methods have been used to model instrument behavior.

\subsection{Instrument Modeling}

A variety of methods and different techniques have been used to govern the behavior of the instrument in a certain environment. ${ }^{44-47,48}$ The following provides an overview of techniques and applied equations and discusses the strengths and weaknesses of each.

\subsubsection{Finite-element method}

Finite-element method (FEM) is a common numerical technique to model a deformable object, ${ }^{47-50}$ including the behavior of the guidewire and catheter inside the body. ${ }^{5,15,16,20-22,24-28,31,32,34,37,39,41,51-69}$ In this method, the instrument is first divided into a set of basic elements connected by nodes. A function that solves the equilibrium equations is found for each element. The equations incorporate the geometry and material information of the instrument. There are different ways to solve these equations. In Refs. 5, 15, 22, 24-28, 37, 54-66, and 69, the instrument is considered as a rod-like structure, a long and thin circular structure with the length being much larger than the diameter. For rod modeling, there are different choices such as Euler-Bernoulli beam theory (deformation due to bending), Kirchhoff rod, ${ }^{15,22,24,26,55}$ which is the geometrically nonlinear generalization of the Euler-Bernoulli beam theory, ${ }^{70}$ Timoshenko beam theory (deformation due to bending and shear), and Cosserat rod, ${ }^{25,27,28,58,61,64-66,69}$ which is the geometrically nonlinear generalization of the Timoshenko beam theory. ${ }^{70}$ In Refs. 15, 22, 37, 41, and 61, the position of the instrument is expressed based on the principles of energy minimization. Thus, the energy function is expressed as 


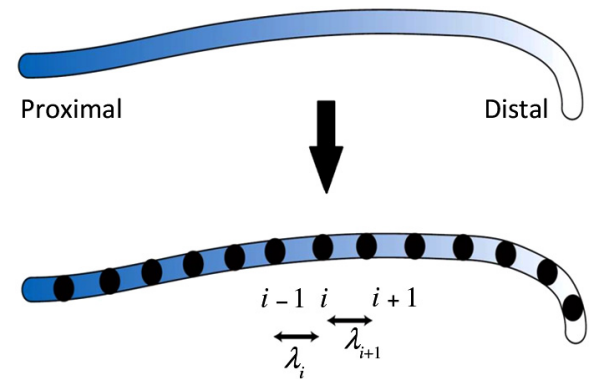

Fig. 2 Discretization of the instrument into small segments; $\lambda_{i}$ and $\lambda_{i+1}$ are not necessarily of the same length.

$E(\mathrm{de})=\min \left[E_{\mathrm{int}}(\mathrm{de})+E_{\mathrm{ext}}(\mathrm{de})\right]$,

where de is the deformation, $E_{\text {int }}$ is the internal energy associated with the flexibility of the instrument, and $E_{\text {ext }}$ is the external energy associated with the applied forces. To solve Eq. (1), the instrument is discretized into multiple segments (see Fig. 2), and the equation is applied to each segment.

FEM is widely used in simulation in different fields because of its numerical stability. Applying this method to model the guidewire and catheter requires a very high computational effort due to the nonlinear underlying effects of FEM. ${ }^{67,68}$ However, the computational time is highly important and especially in some cases, such as training, being real time is necessary.

\subsubsection{Mass-spring model}

In this method, the instrument is considered as a network of masses connected to each other by springs/dampers (Fig. 3). ${ }^{12,23,44,45,71-74}$ The springs not only give flexibility to the model but also constrain the distance between masses. Thus, the number of springs influences the behavior of the model. ${ }^{74}$

The deformable properties of the instrument depend on the parameters of the masses, springs, and dampers as follows:

$m \ddot{x}=k\left(x_{0}-x\right)-d \dot{x}$,

where $m$ is the mass of the particle, $k$ is the spring constant related to the stiffness of the instrument, $d$ is the damping coefficient related to the viscous behavior of the instrument, $x_{0}$ is the rest position of the mass, and $x$ is the current position. Thus, concatenating Eq. (2) of all individual masses $(N)$ into a single $3 N$-dimensional vector and solving them results in the solution for the entire system.

The main advantage of this method is its relative simplicity compared to FEM. However, it is more suited for modeling soft tissue behavior (e.g., the abdominal skin or muscles). In case of a more rigid object, such as the guidewire and catheter, it requires a high computational power, which is against the realtime requirements. Moreover, it is not necessarily accurate, and there is also a risk of numerical instability. ${ }^{45,49}$

\subsubsection{Rigid multibody links}

In this method, the instrument is discretized into a set of rigid bodies connected by massless springs and dampers (Fig. 4). The stiffness and damping coefficients are selected based on the material properties of the segments. ${ }^{75}$

In Refs. 13, 18, 29, 38, 40, 76, 77-80, 81, and 82, the instrument is modeled as rigid bodies connected to their neighbors by joints, and the Newton-Euler equations are used to describe the

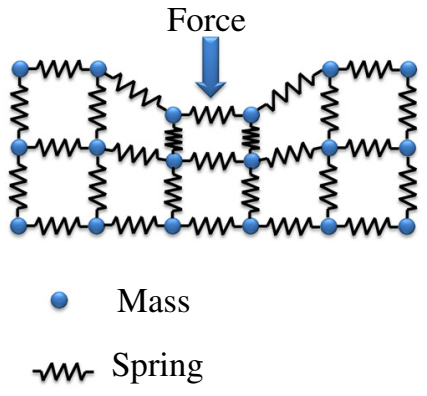

Fig. 3 Mass-spring model.

translational and rotational dynamics. ${ }^{38,76,77,80,83}$ Since the speed of propagating the instrument is slow, the Newton-Euler equations are typically simplified by neglecting inertia and centrifugal force.

In contrast to mass-spring model (MSM), in this method, the length of each segment might be different. Particularly, in guidewire or catheter modeling, it is possible to have shorter segments in the distal side because of more flexibility and longer ones in the proximal side due to more stiffness. This will result in less computational time compared to MSM. Another advantage of this method is that because of its simple structure, it is easy to understand and interpret the results. Moreover, it is relatively easy to incorporate other phenomena such as friction and/or material properties to each individual segment. ${ }^{43}$ On the other hand, the disadvantage of this method is that even though differently sized segment lengths are possible, the simulation is limited to a maximum number of segments, and otherwise it will run into problems.

\subsubsection{Hybrid models}

The mechanical properties of a guidewire/catheter change along the length, more flexibility at the distal side and more stiffness at the proximal side. Due to this property, some studies came with the idea of applying hybrid models, which means using either a combination of different techniques to model different parts of the instrument ${ }^{10,11,28,74,84-67}$ or a new approach that was inspired by different models. ${ }^{9,87-90,91,92,93}$ In this way, they endeavored to make the simulation computationally more efficient.

In Ref. 28, the Cosserat rod model is used for the main body and a rigid multibody approach for the flexible tip. Then, the Lagrangian equations of motion are used to solve the dynamics of both parts (body and tip). In Refs. 84 and 86, the flexible tip and the stiff body are modeled by MSM, separately, after which the connection between them is modeled with an additional rigid link (rigid multibody system). In Refs. 10, 11, and 85, the instrument is discretized into a finite number of flexible multibodies. The deformations of bodies are assumed to be relatively small compared to the displacements. Thus, the segments

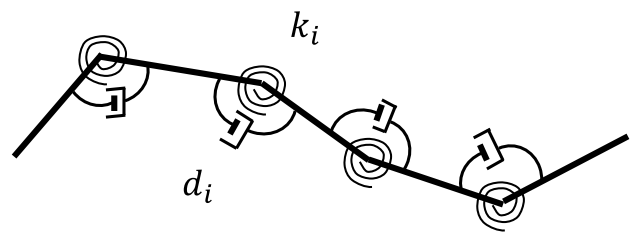

Fig. 4 Multiple rigid bodies connected by joints: $k_{i}$ is the spring constant related to the stiffness and $d_{i}$ is the damping coefficient related to the viscous behavior of joint $i$. 
Table 1 Summary of the reviewed studies.

\begin{tabular}{|c|c|c|c|c|}
\hline \multirow{2}{*}{\multicolumn{2}{|c|}{ Modeling technique }} & \multicolumn{3}{|c|}{ Purpose of the model } \\
\hline & & Training & Preintervention planning & Designing \\
\hline \multirow[t]{4}{*}{ FEM } & Kirchhoff rod theory & References $15,22,24,26$, and 55 & & \\
\hline & Cosserat rod theory & References $25,27,58,61,65$, and 69 & Reference 61 & Reference 61 \\
\hline & Energy minimization & References $15,22,54$, and 61 & References 41 and 37 & Reference 37 \\
\hline & Others & References $16,21,32$, and 34 & References 16 and 39 & References 16 and 43 \\
\hline \multicolumn{2}{|l|}{ MSM } & References $12,23,45$, and $71-73$ & & \\
\hline \multicolumn{2}{|c|}{ Rigid multibody links } & References $13,18,19,29,77,79$, and 81 & $\begin{array}{l}\text { References } 38,40,78,80 \text {, } \\
\text { and } 82\end{array}$ & References 38 and 43 \\
\hline \multicolumn{2}{|c|}{ Hybrid } & $\begin{array}{l}\text { References } 9-11,17,20,28,30,87-90, \\
84,86 \text {, and } 92\end{array}$ & $\begin{array}{l}\text { References } 10,11,17,20 \\
85,91, \text { and } 93\end{array}$ & $\begin{array}{l}\text { References } 9-11,17 \\
20, \text { and } 85\end{array}$ \\
\hline
\end{tabular}

of the instrument are treated as rigid bodies, and displacements are handled by the multibody dynamics approach. Finally, the deformations at their equilibrium position are found by applying FEM.

In Refs. 9, 30, 87-90, 91, 92, and 93, the principles of energy minimization are used to predict the path of the instrument. In contrast to FEM, analytical approximation is applied to solve the optimization problem. In Refs. 9 and 87-90, Hooke's law ${ }^{94}$ is used as the basis for the modeling. In Refs. 91 and 93, a graphbased modeling is described to find the optimal path for the guidewire in different vascular geometries. Table 1 includes a summary of reviewed models.

\subsection{Vessel-Instrument Interaction}

The orientation of the instrument is the result of interaction with the vascular wall and is mainly dominated by the forces experienced during propagation. These forces include the manipulation forces, contact forces with the vascular wall, and frictional forces. In this section, our focus is on the contact and frictional forces.

\subsubsection{Collision}

During the propagation, if the normal distance between the instrument and the vessel is smaller than zero, collision has occurred. Detecting this intersection is referred to as collision detection.

To detect the collision, some studies ${ }^{10,15,37,41,73,83,54}$ considered a circular cross section for the vessel, in which the radii might vary. Therefore, the shape of the vessel is defined by its centerline and its radius, ${ }^{95}$ and the distance between the instrument and the centerline of the vessel is calculated as follows:

$D=d_{i}-\left(R_{v}-R_{G}\right)$,

where $d_{i}$ is the nearest point to the centerline of the vessel, and $R_{V}$ and $R_{G}$ are the radii of the vessel and the instrument, respectively (Fig. 5). If $D \geq 0$, a contact has been occurred.

In Refs. 10, 16, 17, 37, and 55, the vessel is assumed to be rigid, and no deformation occurs due to the contact. Thus, $D$ is used to calculate the normal force based on Hooke's law. ${ }^{94}$ In
Refs. 83 and 92, vessel deformation is not neglected, and an extra term regarding the reaction force from deformation of the wall in radial direction is considered.

In another collision detection approach, an object is approximated by bounding volumes, and instead of the original object, the intersections of bounding volumes are detected. This method is widely used in simulations. ${ }^{45}$ In Refs. 28, 37, 54, 64, 66, 85, 81 , and 92 , the axis-aligned bounding boxes method is used, calculating three-dimensional (3-D) boxes that bound the object and using them to test for collision instead of the original object. In Refs. 23 and 55, the object is bounded by spheres instead of boxes. The advantage of this method is less complexity of collision detection and, thus, less computation time. On the other hand, the accuracy depends on the bounding volumes' size. ${ }^{45}$

\subsubsection{Friction}

During the propagation of an instrument, friction with the vascular wall influences its orientation ${ }^{96}$ and provides force feedback to the user. For the sake of realism of the simulation, modeling the friction is important. However, the coefficient of the friction is not known from the manufacturers and it is determined empirically. There are two forms of friction: kinetic (or sliding) and static. In Refs. 28, 87-90, 55, 82, and 92, the simulation is based on a quasistatic approach. Therefore, the velocities and accelerations of the instrument in the vessel are small, and the velocity-dependent friction forces are neglected. Although in Refs. 13, 77, 96, and 97 both types of the friction are considered, they did not discuss if a higher accuracy was achieved. Some studies ${ }^{9,16,27,33,38,41}$ ignored the friction to tradeoff the realism against computation time. However, in reality,

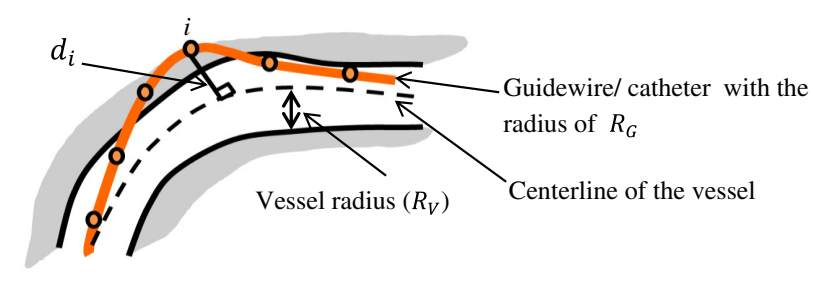

Fig. 5 Collision detection. 
Table 2 Example of commercially available systems.

\begin{tabular}{|c|c|c|c|c|c|}
\hline Device/manufacture & $\begin{array}{l}\text { Modeling } \\
\text { technique }\end{array}$ & Purpose & Application & $\begin{array}{l}\text { Vessel-instrument } \\
\text { interaction }\end{array}$ & Validation method \\
\hline $\begin{array}{l}\text { CathSim, HT Medical } \\
\text { Systems } \text { s }^{18,19,48}\end{array}$ & $\begin{array}{l}\text { Rigid multibody } \\
\text { links }\end{array}$ & Training & $\begin{array}{l}\text { Interventional radiology, } \\
\text { and peripheral intravenous }\end{array}$ & $\begin{array}{l}\text { No available } \\
\text { information }\end{array}$ & $\begin{array}{l}\text { No available } \\
\text { information }\end{array}$ \\
\hline da Vinci ${ }^{16}$ & FEM & $\begin{array}{l}\text { Training, preintervention } \\
\text { planning, and designing }\end{array}$ & Interventional radiology & $\begin{array}{l}\text { Rigid vessel } \\
\text { wall } \\
\text { No friction }\end{array}$ & $\begin{array}{l}\text { Face validity } \\
\text { (clinical validation) }\end{array}$ \\
\hline ICTS/VIST ${ }^{13}$ & $\begin{array}{l}\text { Rigid multibody } \\
\text { links }\end{array}$ & Training & Cardiology & $\begin{array}{l}\text { Blood flow } \\
\text { Friction }\end{array}$ & $\begin{array}{l}\text { No validation } \\
\text { based on Ref. } 13\end{array}$ \\
\hline ICard $^{10,17}$ & Hybrid $^{a}$ & $\begin{array}{l}\text { Training, preintervention } \\
\text { planning, and designing }\end{array}$ & Cardiology & $\begin{array}{l}\text { Rigid vessel } \\
\text { wall }\end{array}$ & $\begin{array}{l}\text { Face validity } \\
\text { (clinical validation) }\end{array}$ \\
\hline Neuro Cath ${ }^{11,20}$ & Hybrid $^{a}$ & $\begin{array}{l}\text { Training, preintervention } \\
\text { planning, and designing }\end{array}$ & $\begin{array}{l}\text { Neuroradiological } \\
\text { procedures }\end{array}$ & Blood flow & $\begin{array}{l}\text { Face validity } \\
\text { (clinical validation) }\end{array}$ \\
\hline Cathl $^{21}$ & FEM & Training & $\begin{array}{l}\text { Endovascular } \\
\text { intervention }\end{array}$ & Rigid vessel wall & $\begin{array}{l}\text { Face validity } \\
\text { (clinical validation) }\end{array}$ \\
\hline
\end{tabular}

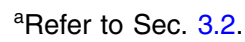

friction is not zero, and the instrument that encounters friction results in a different path in the vascular system. ${ }^{89,96}$

\subsubsection{Blood flow}

Modeling blood flow can be useful to distinguish between a normal and a narrowed vessel. ${ }^{34}$ However, in most of the studies on guidewire and catheter modeling, the effect of blood flow is neglected to reduce the complexity and only a few studies considered it. ${ }^{11,13,20,79,81}$ Considering the blood flow when designing catheters with a side hole for the drug delivery might be interesting as the flow condition can affect the injection procedure. ${ }^{98}$ Moreover, in the presence of vascular malformations, modeling the blood flow might provide a better understanding of the pathological conditions. ${ }^{13,20}$

\subsection{Validation and Evaluation}

The accuracy of any developed model needs to be evaluated. One way to validate a model is by letting a specialist try it out and judge the outcome based on his or her real experiences (face validity). ${ }^{99}$ Most of the reviewed studies validated the simulation results by comparing them with experimental results in phantoms. Phantoms are used both for training and experimental validation. For validation purposes, most of the studies use custom-made phantoms. To fabricate such a phantom, first, they need to extract the vascular geometry in the area of the interest. Thus, Digital Imaging and Communications in Medicine data are obtained via different medical imaging techniques, such as magnetic resonance angiography ${ }^{15,100}$ and computed tomography. ${ }^{28,30,37,54,85}$ Then, a variety of segmentation techniques are used to extract the required information. Next, a cast is constructed based on the extracted data. Recently, 3-D-printing has been used to manufacture phantoms. ${ }^{101}$ Different materials can be used to fabricate the phantom model. For example, to test a guidewire or a catheter behavior, phantom's materials used in the literature include polyvinyl alcohol (PVA), ${ }^{102}$ PVA-hydrogel
(PVA-H), ${ }^{38,80,103}$ PVA-H and silicone (high transparency), ${ }^{104}$ and PVA-cryogel. ${ }^{105}$

Table 2 includes a summary of some commercially available systems with their applications.

\section{Discussion and Conclusion}

This paper has reviewed the existing computer models for both guidewires and catheters navigation in the body. The purposes of these models are categorized in three main groups: (1) training, (2) preintervention planning, and (3) designing instruments. The main techniques used in the modeling are FEM, MSM, and rigid multibody links. In addition, some studies applied different techniques in different parts of the instrument and introduced hybrid methods. FEM is widely used in simulation in different fields because of its numerical stability. However, due to the nonlinear underlying effects, applying FEM to model the guidewire and catheter requires a very high computational effort. Though MSM is easier than FEM to implement, it is more suited for modeling soft tissue behavior (e.g., the abdominal skin or muscles); modeling a more rigid object, such as the guidewire and catheter, requires high computational power. The rigid multibody links technique has a simple structure that makes it easy to understand and interpret the results and relatively faster than the first two methods. Moreover, adding other phenomena, such as friction and/or material properties, to each individual segment is easy. Therefore, for real-time purposes such as training, the first two methods (FEM and MSM) are not suitable, but for preintervention planning and designing purposes, the FEM and MSM are suitable as well as the rigid multibody links technique.

Capturing all details in one model is a hard task. Thus, each study has made the choice to model only the relevant details for their purpose. One advantage of this selection is that it reduces the computational complexity. However, the results might be biased toward the selected details. Moreover, due to the complexity of the interaction with vessels, different studies have made different assumptions, and therefore, they had to make compromises. For example, in most of the research, the 
modeling is based on quasistatic mechanics, which is acceptable as the loading of these instruments is slow and inertial effects can be ignored. Some studies assumed a perfect torque control (the torsion coefficient is considered to be infinite). This feature is taken into account in the design of these instruments, and the assumption is close enough to reality. Furthermore, as the manufacturers do not provide information about the mechanical properties of the instruments, this information is determined empirically. Likewise, vessel properties such as diameter, wall thickness, and stiffness, are determined empirically. In many studies, the vessel wall is assumed to be rigid with a circular cross section; thus, deformation of the vessel is not considered. However, a stiff instrument might cause deformation in the vessel. Additionally, the cross section of the vessels might change due to vascular diseases. Therefore, more studies are required to consider different cross sections and to investigate the deformations especially around the tip of the instrument. Validation is the final step in evaluating the accuracy and effectiveness of a model. Face validity, which is done by letting a specialist try it out and judge the outcome based on his or her real experiences, is a reliable method to test a model. However, in practice, most of the reviewed studies have validated their model by comparing the results with phantom experiment results and some of them did not perform any validation. Further, the few reported validation studies that do exist use inconsistent experimental methodologies. Thus, the validation step is a very important one that needs more focus.

The knowledge provided in this review can help to determine a modeling technique for the instrument, which satisfies the necessary requirements for a particular application.

\section{Disclosures}

All authors declare that they have no conflicts of interest.

\section{Acknowledgments}

This work was part of the research program CONNECT project (Grant No. 12705) within the research program interactive Multi-Interventional Tools that is supported by the Dutch Technology Foundation STW, which is part of the Netherlands Organization for Scientific Research (NWO).

\section{References}

1. J. Dankelman, C. A. Grimbergen, and H. G. Stassen, Engineering for Patient Safety: Issues in Minimally Invasive Procedures, CRC Press, Boca Raton, Florida (2004).

2. J. A. Kaufman and M. J. Lee, Vascular and Interventional Radiology, Elsevier Health Sciences, Amsterdam, Netherlands (2013).

3. L. Aklog et al., "Techniques and results of direct-access minimally invasive mitral valve surgery: a paradigm for the future," $J$. Thorac. Cardiovasc. Surg. 116(5), 705-715 (1998).

4. J. M. Lasala and J. H. Rogers, Interventional Procedures for Adult Structural Heart Disease: Expert Consult-Online, Elsevier Health Sciences, Amsterdam, Netherlands (2013).

5. I. Badash et al., "Innovations in surgery simulation: a review of past, current and future techniques," Ann. Transl. Med. 4(23), 453 (2016).

6. A. M. Al-Moghairi and H. S. Al-Amri, "Management of retained intervention guide-wire: a literature review," Curr. Cardiol. Rev. 9(3), 260266 (2013).

7. K. Kunkler, "The role of medical simulation: an overview," Int. J. Med. Rob. Comput. Assisted Surg. 2(3), 203-210 (2006).

8. R. Aggarwal et al., "Virtual reality simulation training can improve inexperienced surgeons' endovascular skills," Eur. J. Vasc. Endovasc. Surg. 31(6), 588-593 (2006).
9. M. K. Konings et al., "Analytical guide wire motion algorithm for simulation of endovascular interventions," Med. Biol. Eng. Comput. 41(6), 689-700 (2003).

10. Y. Wang et al., "Real-time interactive simulator for percutaneous coronary revascularization procedures," Comput. Aided Surg. 3(5), 211227 (1998).

11. Z. Li et al., "Computer environment for interventional neuroradiology procedures," Simul. Gaming 32(3), 404-419 (2001).

12. C. Basdogan, C.-H. Ho, and M. A. Srinivasan, "Virtual environments for medical training: graphical and haptic simulation of laparoscopic common bile duct exploration," IEEE/ASME Trans. Mechatron. 6(3), 269-285 (2001).

13. S. Cotin et al., "ICTS, an interventional cardiology training system," Stud. Health Technol. Inf. 70, 59-65 (2000).

14. D. W. Shaffer et al., "Design principles for the use of simulation as an aid in interventional cardiology training," Minimally Invasive Ther. Allied Technol. 10(2), 75-82 (2001).

15. $\mathrm{S}$. Li et al., "A catheterization-training simulator based on a fast multigrid solver," IEEE Comput. Graphics Appl. 32(6), 56-70 (2012).

16. J. H. Anderson et al., "daVinci-a vascular catheterization simulator," J. Vasc. Interventional Radiol. 8(1), 261 (1997).

17. C. Chui et al., "Icard: an interventional cardiology simulator for percutaneous coronary revascularisation," in Computer Assisted Radiology and Surgery (CAR), Tokyo (1998).

18. D. Meglan, "Making surgical simulation real," ACM SIGGRAPH Comput. Graphics 30(4), 37-39 (1996).

19. M. Ursino et al., "CathSim ${ }^{\mathrm{TM}}$ : an intravascular catheterization simulator on a PC," Stud. Health Technol. Inf. 62, 360-366 (1998).

20. W. L. Nowinski and C.-K. Chui, "Simulation of interventional neuroradiology procedures," in Int. Workshop on Medical Imaging and Augmented Reality, Proc., pp. 87-94, IEEE (2001).

21. P. Rebholz et al., "CathI-training system for PTCA. A step closer to reality," in Medical Simulation, S. Cotin and D. Metaxas, Eds., pp. 249-255, Springer, Berlin, Heidelberg (2004).

22. S. Li et al., "A novel FEM-based numerical solver for interactive catheter simulation in virtual catheterization," Int. J. Biomed. Imaging 2011, 1-8 (2011).

23. F. Wang et al., "A computer-based real-time simulation of interventional radiology," in 29th Annual Int. Conf. of the IEEE Engineering in Medicine and Biology Society, pp. 1742-1745, IEEE (2007).

24. M. Luo et al., "A robust and real-time vascular intervention simulation based on Kirchhoff elastic rod," Comput. Med. Imaging Graphics 38(8), 735-743 (2014).

25. L. Duratti et al., "A real-time simulator for interventional radiology," in Proc. of the ACM Symp. on Virtual Reality Software and Technology, pp. 105-108, ACM (2008).

26. C. Zhou et al., "Cardiovascular-interventional-surgery virtual training platform and its preliminary evaluation," Int. J. Med. Rob. Comput. Assisted Surg. 11(3), 375-387 (2015).

27. P. Korzeniowski et al., "Simulation of catheters and guidewires for cardiovascular interventions using an inextensible Cosserat rod," Lect. Notes Comput. Sci. 8789, 112-121 (2014).

28. W. Tang et al., "A stable and real-time nonlinear elastic approach to simulating guidewire and catheter insertions based on Cosserat rod," IEEE Trans. Biomed. Eng. 59(8), 2211-2218 (2012).

29. Y. Wang et al., "A virtual-reality simulator and force sensation combined catheter operation training system and its preliminary evaluation," Int. J. Med. Rob. Comput. Assisted Surg. 13, e1769 (2017).

30. D. Zhang et al., "Vascular deformation for vascular interventional surgery simulation," Int. J. Med. Rob. Comput. Assisted Surg. 6(2), 171177 (2010).

31. J. Guo et al., "Virtual reality simulators based on a novel robotic catheter operating system for training in minimally invasive surgery," J. Rob. Mechatronics 24(4), 649-655 (2012).

32. $\mathrm{X}$. Wu et al., "New approaches to computer-based interventional neuroradiology training," Stud. Health Technol. Inf. 111, 602-607 (2005).

33. A. Alaraj et al., "Virtual reality training in neurosurgery: review of current status and future applications," Surg. Neurol. Int. 2, 52 (2011).

34. J. Anderson et al., "Virtual reality training in interventional radiology: the Johns Hopkins and Kent Ridge digital laboratory experience," Semin. Interventional Radiol. 19(2), 179-186 (2002). 
35. J. Gosai, M. Purva, and J. Gunn, "Simulation in cardiology: state of the art," Eur. Heart J. 36(13), 777-783 (2015).

36. W. Voelker et al., "Does simulation-based training improve procedural skills of beginners in interventional cardiology? - a stratified randomized study," J. Interventional Cardiol. 29(1), 75-82 (2016).

37. W. Lawton et al., "Tubes in tubes: catheter navigation in blood vessels and its applications," Int. J. Solids Struct. 37(22), 3031-3054 (2000).

38. K. Takashima et al., "Numerical analysis and experimental observation of guidewire motion in a blood vessel model," Med. Eng. Phys. 36(12), 1672-1683 (2014).

39. S. Bhat, T. Kesavadas, and K. R. Hoffmann, "A physically-based model for guidewire simulation on patient-specific data," Int. Congr. Ser. 1281, 479-484 (2005).

40. V. Guilloux et al., "Simulation of guidewire navigation in complex vascular structures," Proc. SPIE 6141, 614107 (2006).

41. F. M. Cardoso and S. S. Furuie, "Guidewire path determination for intravascular applications," Comput. Meth. Biomech. Biomed. Eng. 19(6), 628-638 (2016).

42. R. W. Martin and C. C. Johnson, "Design characteristics for intravascular ultrasonic catheters," Int. J. Cardiac Imaging 4, 201-216 (1989).

43. Y. Y. Cai et al., "Simulation-based virtual prototyping of customized catheterization devices," J. Comput. Inf. Sci. Eng. 4(2), 132-139 (2004).

44. J. Burgner-Kahrs, D. C. Rucker, and H. Choset, "Continuum robots for medical applications: a survey," IEEE Trans. Rob. 31(6), 1261-1280 (2015).

45. L. Torpey, "A virtual environment simulation for guidewire/catheter and blood vessel interaction," $\mathrm{PhD}$ Dissertation, University of Leeds, School of Computing Studies (2010).

46. A. Zorcolo et al., "A volumetric virtual environment for catheter insertion simulation," in Virtual Environments, J. Mulder and R. van Liere, Eds., pp. 125-134, Springer, Vienna (2000).

47. S. F. F. Gibson and B. Mirtich, "A survey of deformable modeling in computer graphics," Mitsubishi Electric Information Technology Center America (1997).

48. M. Bro-Nielsen, "Simulation techniques for minimally invasive surgery," Minimally Invasive Ther. Allied Technol. 6(2), 106-110 (1997).

49. A. Liu et al., "A survey of surgical simulation: applications, technology, and education," Presence 12(6), 599-614 (2003).

50. T. Halic, "Virtual environments and their applications in surgical training," PhD Thesis, University of Arkansas, ProQuest (2008).

51. S. Cotin et al., "New approaches to catheter navigation for interventional radiology simulation," Comput. Aided Surg. 11(6), 300-308 (2006).

52. P. Wei et al., "FEM-based guide wire simulation and interaction for a minimally invasive vascular surgery training system," in Proc. of the 11th World Congress on Intelligent Control and Automation (WCICA) (2014).

53. Z. Qiukui and P. Haigron, "A FEM model for interactive simulation of guide wire navigation in moving vascular structures," in Sixth Int. Conf. on Intelligent Systems Design and Engineering Applications (ISDEA), pp. 13-16, IEEE (2015).

54. N. Becherer et al., "Interactive physical simulation of catheter motion within mayor vessel structures and cavities for ASD/VSD treatment," Proc. SPIE 6509, 65090U (2007).

55. W. Tang et al., "A realistic elastic rod model for real-time simulation of minimally invasive vascular interventions," Visual Comput. 26(9), 1157-1165 (2010).

56. D. Huang et al., "Modeling and simulation of multi-frictional interaction between guidewire and vessel," Lect. Notes Comput. Sci. 9218, 524-537 (2015).

57. P. Chembrammel, H. M. Younus, and T. Kesavadas, "Modelling and simulation of guide-wire interaction with vessel using constrained multibody dynamics," in ASME Int. Mechanical Engineering Congress and Exposition, p. V04AT04A083, American Society of Mechanical Engineers (2013).

58. S. Nuti, A. Ruimi, and J. N. Reddy, "Modeling the dynamics of filaments for medical applications," Int. J. Non Linear Mech. 66, 139148 (2014).

59. T. Liu et al., "Modeling and validation of the three-dimensional deflection of an MRI-compatible magnetically actuated steerable catheter," IEEE Trans. Biomed. Eng. 63(10), 2142-2154 (2016).
60. M. Khoshnam, M. Azizian, and R. V. Patel, "Modeling of a steerable catheter based on beam theory," in IEEE Int. Conf. on Robotics and Automation (ICRA), pp. 4681-4686, IEEE (2012).

61. W. Lawton et al., "Ribbons and groups: a thin rod theory for catheters and filaments," J. Phys. A 32(9), 1709-1735 (1999).

62. H. Zhao et al., "Aspects of contact force and path configuration generated during catheter navigation," in Proc. of the Spring Simulation MultiConf., p. 174, Society for Computer Simulation International (2009).

63. Y. Wang et al., "Real-time simulation of catheterization in endovascular surgeries," Comput. Anim. Virtual Worlds 27(3-4), 185-194 (2016).

64. J. Back et al., "Catheter contact force estimation from shape detection using a real-time Cosserat rod model," in IEEE/RSJ Int. Conf. on Intelligent Robots and Systems (IROS), pp. 2037-2042, IEEE (2015).

65. Z.-J. Gao et al., "Fast and stable guidewire simulator for minimally invasive vascular surgery," in 37th Annual Int. Conf. of the IEEE Engineering in Medicine and Biology Society (EMBC), pp. 58095812, IEEE (2015).

66. D. K. Pai, "STRANDS: interactive simulation of thin solids using Cosserat models," Comput. Graphics Forum 21(3), 347-352 (2002).

67. U. Meier et al., "Real-time deformable models for surgery simulation: a survey," Comput. Meth. Programs Biomed. 77(3), 183-197 (2005).

68. B. Preim and C. P. Botha, Visual Computing for Medicine: Theory, Algorithms, and Applications, Chapter 21, pp. 154-156, Newnes, Oxford and Boston (2013).

69. X.-R. Cheng et al., "A fast and stable guidewire model for minimally invasive vascular surgery based on Lagrange multipliers," in Seventh Int. Conf. on Information Science and Technology (ICIST), pp. 109114, IEEE (2017).

70. H. Lang, J. Linn, and M. Arnold, "Multi-body dynamics simulation of geometrically exact Cosserat rods," Multibody Sys. Dyn. 25(3), 285312 (2011).

71. V. Luboza et al., "Simulation of endovascular guidewire behavior and experimental validation," Comput. Meth. Biomech. Biomed. Eng. 14(6), 515-520 (2011).

72. V. Luboz et al., "Real-time guidewire simulation in complex vascular models," Visual Comput. 25(9), 827-834 (2009).

73. V. Luboz et al., "ImaGiNe Seldinger: first simulator for Seldinger technique and angiography training," Comput. Methods Programs Biomed. 111(2), 419-434 (2013).

74. S. Cotin, "Computer based interactive medical simulation," $\mathrm{PhD}$ Dissertation, Université des Sciences et Technologie de Lille-Lille I (2008).

75. W. Ding, Self-Excited Vibration: Theory, Paradigms, and Research Methods, pp 374-375, Springer Science and Business Media, Berlin/Heidelberg, Germany (2013).

76. D. E. Stewart, "Rigid-body dynamics with friction and impact," Soc. Ind. Appl. Math. 42(1), 3-39 (2000).

77. K. Ikuta et al., "Portable virtual endoscope system with force and visual display for insertion training," in Proc. of the IEEE/RSJ Int. Conf. on Intelligent Robots and Systems (IROS), Vol. 1, pp. 720-726 (2000).

78. M. Kukuk, "A model-based approach to intra operative guidance of flexible endoscopy," PhD Thesis, Princeton (2002).

79. S. L. Dawson et al., "Equipment and technology—designing a computer-based simulator for interventional cardiology training," Catheterization Cardiovasc. Interventions 51(4), 522-527 (2000).

80. K. Takashima et al., "Evaluation of the effect of catheter on the guidewire motion in a blood vessel model by physical and numerical simulations," J. Biomech. Sci. Eng. 12(4), 17-00181 (2017).

81. W. Wang et al., "Novel, robust, and efficient guidewire modeling for PCI surgery simulator based on heterogeneous and integrated chainmails," in 14th Int. Conf. on Computer-Aided Design and Computer Graphics (CAD/Graphics), pp. 105-112, IEEE (2015).

82. J. Jung et al., "Model validation and simulation studies: effects of nonlinear internal device friction on continuum robotic manipulators," in IEEE Int. Conf. Robotics and Automation (2012).

83. F. Amirouche, Fundamentals of Multibody Dynamics: Theory and Applications, Birkhäuser, Boston (2006).

84. S.-H. Mi et al., "A multi-body mass-spring model for virtual reality training simulators based on a robotic guide wire operating system," in Proc. of the IEEE Int. Conf. on Robotics and Biomimetics (ROBIO), Shenzhen (2013). 
85. Y. P. Wang et al., "Topology supported finite element method analysis of catheter/guidewire navigation in reconstructed coronary arteries," in Computers in Cardiology, Vol. 24, IEEE (1997).

86. S.-H. Mi et al., "A collision response algorithm for 3D virtual reality minimally invasive surgery simulator," in 26th Chinese Control and Decision Conf. (CCDC), pp. 4594-4599, IEEE (2014).

87. T. Alderliesten, M. K. Konings, and W. J. Niessen, "Simulation of minimally invasive vascular interventions for training purposes," Comput. Aided Surg. 9(1-2), 3-15 (2004).

88. T. Alderliesten, P. A. Bosman, and W. J. Niessen, "Towards a real-time minimally-invasive vascular intervention simulation system," IEEE Trans. Med. Imaging 26(1), 128-132 (2007).

89. T. Alderliesten, M. K. Konings, and W. J. Niessen, "Modeling friction, intrinsic curvature, and rotation of guide wires for simulation of minimally invasive vascular interventions," IEEE Trans. Biomed. Eng. 54(1), 29-38 (2007).

90. T. Alderliesten, M. K. Konings, and W. J. Niessen, "Robustness and complexity of a minimally invasive vascular intervention simulation system," Med. Phys. 33(12), 4758-4769 (2006).

91. S. Schafer et al., "Planning image-guided endovascular interventions: guidewire simulation using shortest path algorithms," Proc. SPIE 6509, 65092C (2007).

92. P. T. Tran et al., "Position control of robotic catheters inside the vessel based on a predictive minimum energy model," in IEEE Int. Conf. on Systems, Man, and Cybernetics (SMC), pp. 004687-004693, IEEE (2016).

93. L. Xu et al., "An improved endovascular guidewire position simulation algorithm," in 9th IEEE Int. Symp. on Biomedical Imaging (ISBI), pp. 1196-1199, IEEE (2012).

94. G. Arfken, Mathematical Methods for Physicists, Academic Press, Inc., San Diego, California (1985).

95. M. Schaap et al., "Standardized evaluation methodology and reference database for evaluating coronary artery centerline extraction algorithms," Med. Image Anal. 13(5), 701-714 (2009).

96. K. Takashima et al., "Contact and friction between catheter and blood vessel," Tribol. Int. 40(2), 319-328 (2007).

97. W. Ahn et al., "Model of frictional contact with soft tissue for colonoscopy simulator," in IEEE Int. Conf. on Systems, Man and Cybernetics, Vol. 3, pp. 2714-2719, IEEE (2005).

98. R. Mongrain et al., "Study of catheter designs and drug mixing processes using 2D steady numerical simulations," Med. Biol. Eng. Comput. 37(1), 64-70 (1999).

99. F. J. Carter et al., "Consensus guidelines for validation of virtual reality surgical simulators," Surg. Endosc. Other Interventional Tech. 19(12), 1523-1532 (2005).

100. P. M. O'Flynn, E. T. Roche, and A. S. Pandit, "Generating an ex vivo vascular model," ASAIO J. 51(4), 426-433 (2005).

101. C. N. Ionita et al., "Challenges and limitations of patient-specific vascular phantom fabrication using 3D Polyjet printing," Proc. SPIE 9038, 90380M (2014).
102. K. Funamoto, O. Yamashita, and T. Hayase, "Poly (vinyl alcohol) gel ultrasound phantom with durability and visibility of internal flow," J. Med. Ultrason. 42(1), 17-23 (2015).

103. Y. U. ChangHo et al., "Development of an in vitro tracking system with poly (vinyl alcohol) hydrogel for catheter motion," J. Biomech. Sci. Eng. 5(1), 11-17 (2010).

104. C.-H. Yu et al., "Comparative analysis for evaluating the traceability of interventional devices using blood vessel phantom models made of PVA-H or silicone," Technol. Health Care 23(s2), S301-S310 (2015).

105. K. J. M. Surry et al., "Poly (vinyl alcohol) cryogel phantoms for use in ultrasound and MR imaging," Phys. Med. Biol. 49(24), 5529-5546 (2004).

Hoda Sharei received her BSc degree in biomedical engineering from Amirkabir University of Tehran, Iran, in 2008 and her MSc degree in electrical engineering from Shiraz University, Iran, in 2012. After that, she was a researcher at Liege University, Belgium for one year. Since July 2014, she is pursuing her PhD in the Department of BioMechanical Engineering, Faculty of Mechanical, Maritime, and Materials Engineering (3mE), Delft University of Technology, Delft, the Netherlands.

Tanja Alderliesten received her BSc and MSc degrees in medical computer science from Utrecht University, Utrecht, the Netherlands (cum laude). In her PhD, she focused on "simulation of minimally invasive vascular interventions for training purposes" at the Image Sciences Institute, Faculty of Medicine, Utrecht University, Utrecht. Currently, she is a senior researcher in the Department of Radiation Oncology, Academic Medical Center, University of Amsterdam, the Netherlands.

John J. van den Dobbelsteen obtained his PhD from the Department of Physiology, Erasmus MC (Medical Center), Rotterdam. Since 2005, he joined the Department of BioMechanical Engineering, Faculty of $3 \mathrm{mE}$, Delft University of Technology as a postdoc researcher, and currently, he is an associate professor at Delft University of Technology, Delft, the Netherlands.

Jenny Dankelman is professor in Minimally Invasive Surgery and Interventional Techniques at the Delft University of Technology. In 2001 she was awarded the Antoni van Leeuwenhoek chair and in 2013 she became medical delta professor. Her research group cooperates with several hospitals such as Leiden UMC where she holds a part time professorship position, Erasmus MC Rotterdam and the AMC Amsterdam. Her interests and research projects are in the fields of designing novel medical instruments, haptics, training and simulation systems, and patient safety, with the focus on minimally invasive techniques. 